\title{
Response of Sediment Bacterial Communities to Sudden Vegetation Dieback in a Coastal Wetland
}

\author{
Wade H. Elmer and Peter Thiel, Department of Plant Pathology and Ecology, Connecticut Agricultural Experiment \\ Station, New Haven 06511; and Blaire Steven, Department of Environmental Sciences, Connecticut Agricultural \\ Experiment Station, New Haven 06511
}

Accepted for publication 20 November 2016.

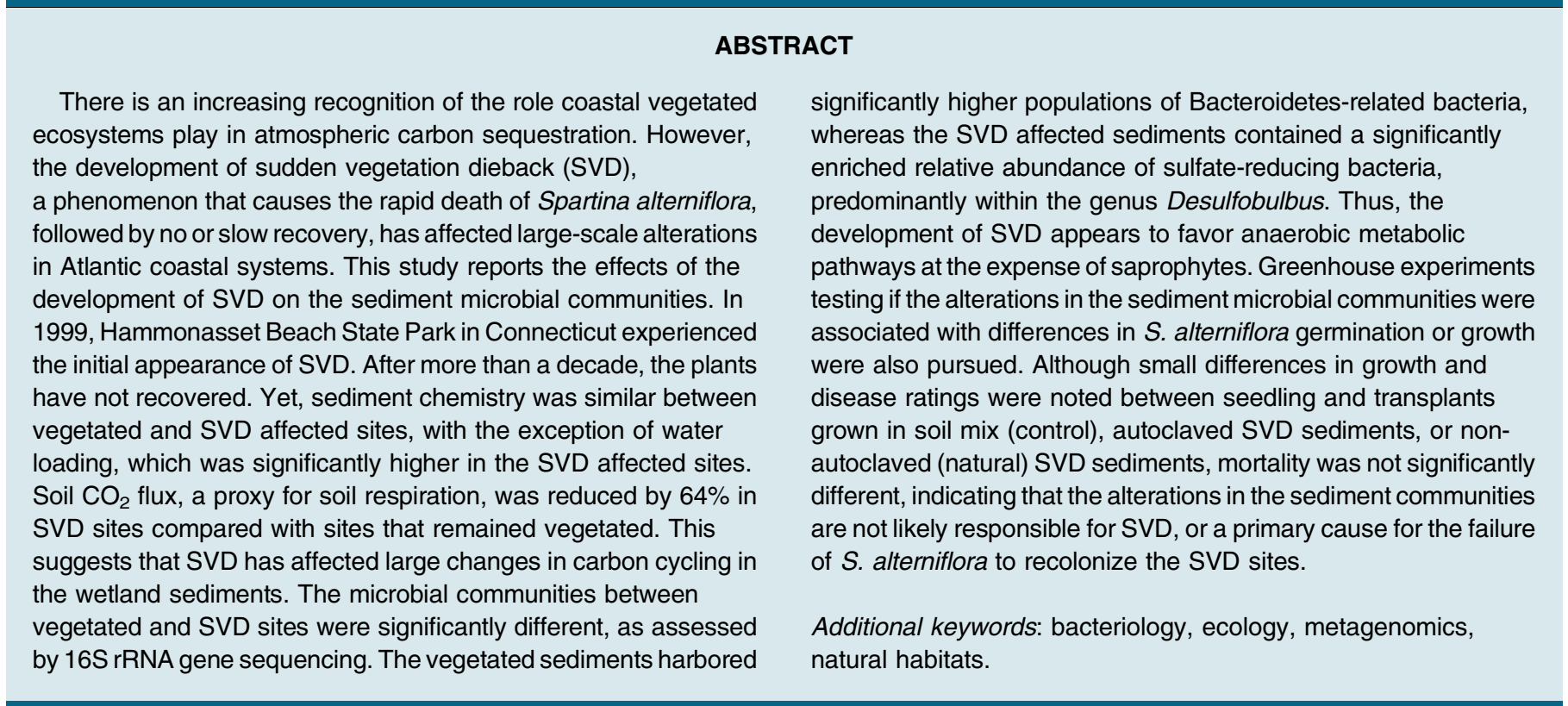

There is an increasing recognition that cutting carbon emissions alone will not likely achieve atmospheric carbon concentrations sufficient to avoid the most drastic consequences of climate change (Lal 2004; Schimel 1995). Another component to mitigating climate change is identifying carbon sinks that can sequester carbon from the atmosphere and store it in a stable form. One potential carbon sink is vegetated coastal ecosystems (i.e., tidal marshes, mangroves, and seagrass meadows) also referred to as "blue carbon" sinks (Herr et al. 2012; Nellemann and Cocoran 2009). Vegetated coastal ecosystems store carbon within living biomass aboveground (e.g., leaves and stems) belowground (roots), and within nonliving biomass (e.g., leaf litter). The potency of coastal

Corresponding author: B. Steven; E-mail address: blaire.steven@ct.gov

*The $\boldsymbol{e}$-Xtra logo stands for "electronic extra" and indicates that two supplementary tables are published online.

(C) 2017 The American Phytopathological Society ecosystems as carbon sinks is partly due to their high primary productivity. Salt marshes are among the most productive ecosystems on the planet, generating approximately $40 \%$ more biomass annually than produced in a similar area of forest (Bertness et al. 2008; Hopkinson et al. 2012). This photosynthetically fixed carbon is eventually passed to the sediments through plant litter, root exudates, or death, becoming the substrate for microbial metabolism (Blodau 2002; Picek et al. 2007). As wetlands are defined by periods of water saturation and a water table close to or at the surface, waterlogged conditions are a fundamental characteristic of coastal wetland sediments. Water acts as a diffusion barrier to oxygen, so flooded soils rapidly become anaerobic. In this regard, a significant fraction of the degradation of plant-derived carbon occurs in anoxic conditions (Mcleod et al. 2011). Because anaerobic degradation of organic matter happens relatively slowly, the rates of organic inputs from the vegetation occurs at a greater rate than losses by microbial respiration, so the net effect is storage of this carbon in wetland sediments. This can result in a substantial store of 
carbon. Seagrass meadows and mangroves have accreted carbonrich sediments $>10 \mathrm{~m}$ thick (Loiacono et al. 2011; McKee et al. 2007). The final mechanism through which coastal systems can store carbon is by sedimentation and trapping of water transported materials from outside their boundaries (i.e., upstream rivers). Up to $50 \%$ of carbon sequestered in seagrass meadows is thought to be of external origin (Kennedy et al. 2010). In this way, blue carbon can be sequestered over the short term in vegetation biomass, and over longer time scales in sediments. Radiocarbon dating of carbon sequestered in coastal sediments indicates that carbon can be stored for hundreds to thousands of years (Brevik and Homburg 2004; Choi and Wang 2004). This large and stable reservoir of blue carbon is driving an increased interest in coastal wetland carbon cycling and the role of coastal wetlands in climate change mitigation.

Salt marshes along the Atlantic and northern Gulf Coasts of temperate North America are commonly characterized by stands of the smooth cordgrass Spartina alterniflora. S. alterniflora is notable for its rates of carbon fixation. Organic carbon loading to wetland sediments by $S$. alterniflora are in the range of 68 to 78 moles per $\mathrm{m}^{2}$ per year, higher than for Scripus or Phragmites dominated wetlands (Howes et al. 1985; Liao et al. 2007). The transfer of photosynthetically fixed carbon from the plant to the sediments happens rapidly. Measurements based on ${ }^{13} \mathrm{CO}_{2}$ labeling indicate that 30 to $55 \%$ of the carbon fixed by photosynthesis is available for soil respiration after just $24 \mathrm{~h}$ (Spivak and Reeve 2015). S. alterniflora is also associated with significantly higher levels of soluble organic carbon and sediment microbial biomass in comparison with other marsh grasses ( $\mathrm{Bu}$ et al. 2015). Taken together, these observations suggest that $S$. alterniflora, and its associated phytobiome, is a potent player in storage of carbon in wetland sediments.

Multiple S. alterniflora salt marshes along the Atlantic and Gulf Coasts of the United States have experienced developments of sudden vegetation dieback (SVD), that presents as an initial thinning and browning of the above-ground foliage with plant death occurring over a period of weeks to months (Alber et al. 2008). A key characteristic of SVD is the death of the propagative rhizomes, which inhibits regrowth, so that SVD affected sites can persist for years (Elmer et al. 2013). The etiology of SVD remains to be explained, although fungal pathogens (Elmer et al. 2012; Elmer 2016), invasive crabs (Schultz et al. 2016), and drought (McKee et al. 2004) have been proposed as the cause, or contributing to the development of SVD. Regional differences or interactions between different factors may play a role in the difficulty in identifying a unified explanation of SVD development (Elmer 2014). Much of the research on SVD has focused on trying to identify the causes of SVD, whereas research into the consequences of SVD on the structure and function of wetland ecosystems has been limited.

In 1999, an occurrence of SVD developed in Hammonasset Beach State Park marshes in Madison, CT. The dieback patches occurred along creek banks dominated by the tall form of $S$. alterniflora and resulted in completely barren patches of wetland sediments, which largely remain unvegetated. SVD and healthy marsh patches are often directly adjacent with no obvious patterns related to hydrology or location (Elmer et al. 2013). In this study, the objectives were to characterize the effects of vegetation loss on the bacterial communities in vegetated and nonvegetated sediments in an SVD affected wetland and to identify the bacteria that may be involved in processing carbon fixed by $S$. alterniflora.

\section{MATERIALS AND METHODS}

Site description and sample collection. Hammonasset Beach State Park is located in Madison, Connecticut on the shore of the

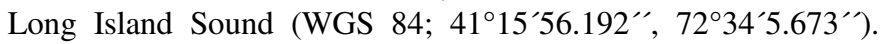
Occurrences of SVD have been documented since at least 1999 , with areas affected by SVD being completely unvegetated and visibly apparent as bare peat. The sites of SVD are generally patchy and occur along creek banks (Fig. 1).

Three distinct wetland sites were identified in the salt marsh that consisted of unvegetated sediments or healthy vegetated patches. Sites were approximately $20 \mathrm{~m}$ apart with two sites on the north side of the creek and one on the south side. From each site, two locations were identified approximately $1 \mathrm{~m}$ apart for two replicate locations per site. Each location was delimited by the $20 \mathrm{~cm}$ soil collar used

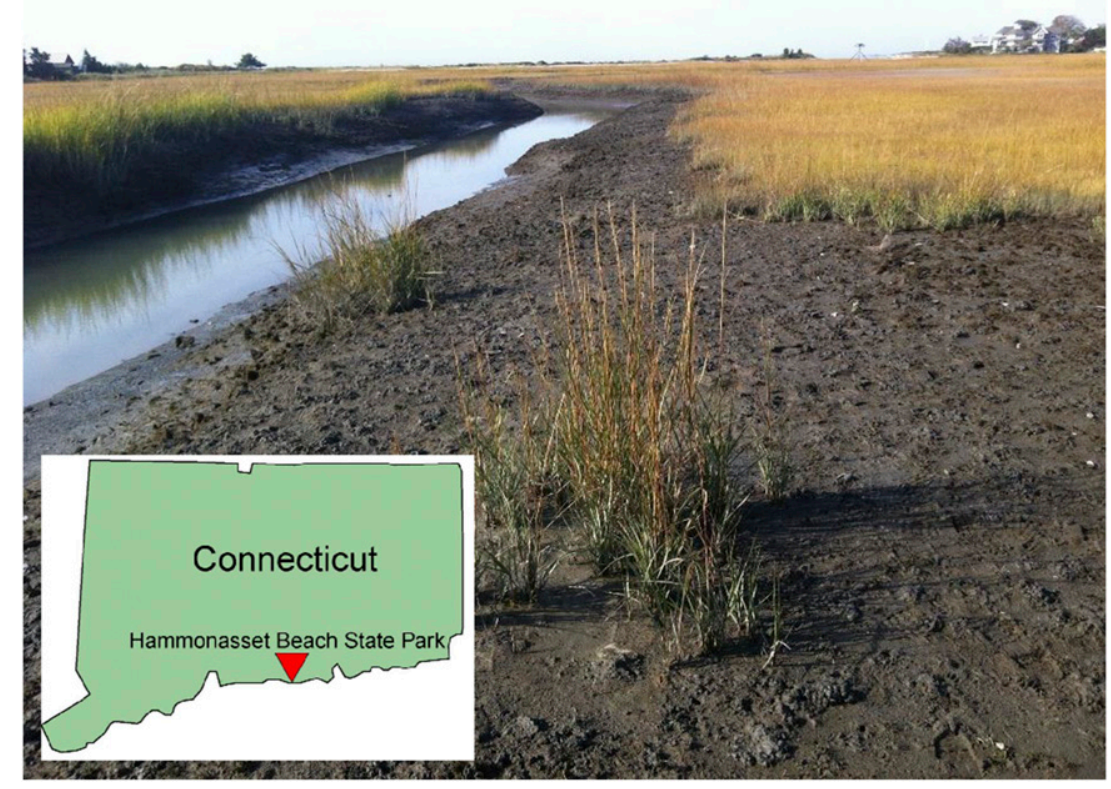

Fig. 1. Field site location and characteristics of a sudden vegetation dieback (SVD) affected wetland. The inset map shows the location of the field site in the state of Connecticut. In the photo foreground, a wetland patch affected by SVD is shown. The sediments are almost completely devoid of the dominant vegetation Spartina alterniflora. Healthy patches of wetland can be seen on the opposite side of the creek where vegetation grows up to the banks. 
for soil $\mathrm{CO}_{2}$ flux measurement (see below). After $\mathrm{CO}_{2}$ flux measurements from each location, triplicate approximate 5-g soil samples were collected for DNA extraction. Thus, this sampling scheme resulted in three replicates per location, two locations per site, and three replicate sites for both SVD and vegetated wetland patches (i.e., 18 biological replicates for each of SVD and vegetated conditions). Sediment samples for DNA extraction were collected on 23 September 2014. Sampling times were selected during low tide to ensure that the soils would not be flooded at the time of sampling. At the time of sampling, the average soil temperature was $20.6^{\circ} \mathrm{C}$.

The sediment samples were collected with a sterile spatula and only the upper $\sim 2 \mathrm{~cm}$ was collected. For the vegetated sites, the upper soil was collected in the vicinity of the plants, but attempting to exclude plant material or roots. The sediment samples for DNA extraction were placed on dry ice and transported back to the Connecticut Agricultural Experiment Station (CAES) where they were stored at $-80^{\circ} \mathrm{C}$ until processing. For soil chemistry, a single approximately 100 -g soil sample was collected from each location and transported on ice to the station where it was stored in the freezer until processing.

Soil $\mathrm{CO}_{2}$ flux and soil chemistry. Soil $\mathrm{CO}_{2}$ flux was measured with the LI-8100A Soil Gas Flux Survey System (LI-COR Biosciences, Lincoln, NE). This involved placing a $20 \mathrm{~cm}$ diameter soil collar into the sediments and determining the collar offset above the surface to accurately determine the chamber head space. The supplied temperature and moisture probes were inserted into the soil adjacent to the collar. Once the survey chamber was placed on the collar soil, flux data were collected for $1 \mathrm{~min}$ and $30 \mathrm{~s}$. After the completion of the measurement, the survey chamber was removed and replaced for a total of three replicate soil $\mathrm{CO}_{2}$ measurements per location.

All soil chemistry measurements were performed by Galbraith Laboratories Inc. (Knoxville, TN). The procedures employed were as follows: carbon and nitrogen, elemental analysis via the LECHO CHN 628 Analyzer; iron and phosphorous, Inductively Coupled Plasma Atomic Emission Spectrometry via the ICP-OES Optima 5300; and sulfur, sulfur analysis via the LECO SC-432DR. Soil moisture was determined gravimetrically by drying the soil samples overnight at $70^{\circ} \mathrm{C}$ and determining the percent mass of water.

Soil DNA extraction and amplification/sequencing of $16 \mathrm{~S}$ rRNA genes. For each of the soil samples collected for DNA extraction, a 0.5-g subsample was aseptically transferred to a Power Bead Tube (MO BIO Power Soil Kit, Carlsbad, CA) and DNA was extracted using the supplied protocols. All DNA extractions were verified by gel electrophoresis in $1 \%$ agarose gels and the DNA products were quantified with a NanoDrop spectrophotometer (NanoDrop Lite, Thermo Scientific, Waltham, MA).

For each PCR reaction for amplification of bacterial 16S rRNA genes, an amount of $1 \mathrm{ng}$ of input DNA was used per reaction. The $16 \mathrm{~S}$ rRNA genes were amplified with the primers 515F (5'-TCG TCG GCA GCG TCA GAT GTG TAT AAG AGA CAG $\overline{\text { GTG }}$ CCA GCM GCC GCG GTA A) and 806R (GTC TCG TGG GCT CGG AGA TGT GTA TAA GAG ACA GGG ACT ACH VGG GTW TCT AAT), which also included Illumina adaptor sequences (indicated by underlining). Cycling conditions were as follows: Initial denaturation $95^{\circ} \mathrm{C}$ for $3 \mathrm{~min} ; 35$ cycles of $95^{\circ} \mathrm{C}$ for $45 \mathrm{~s}, 55^{\circ} \mathrm{C}$ for $60 \mathrm{~s}, 72^{\circ} \mathrm{C}$ for $60 \mathrm{~s}$; a final extension at $72^{\circ} \mathrm{C}$ for $10 \mathrm{~min}$. The approximately 350 -bp PCR products were verified by gel electrophoresis and purified with the QIAquick PCR purification kit (Qiagen). Sequence indexing was performed using the index PCR procedure, employing the Nextera DNA Library Prep Kit (Illumina Inc., San Diego, CA). Following indexing, PCR amplicons were purified as above and pooled in equal molar concentrations $(1 \mu \mathrm{g})$.
The resulting DNA was sequenced on the Illumina HiSeq platform at the Yale Center for Genome Analysis with standard protocols on the HiSeq2500 employing paired end $2 \times 150$ chemistry. Paired end sequences were assembled into contigs using the make.contigs command with default parameters in the mothur (Schloss et al. 2009) software package, only retaining contigs of at least 291 bases in length. Each contig was further screened to remove any sequences with any ambiguous nucleotide calls or homopolymers of $\geq 7$ bases. Potentially chimeric sequences were identified with the mothur implementation of uchime (Edgar et al. 2011). A total of $297,768(6.1 \%)$ sequences were identified as potentially chimeric and removed from further analysis. Sequences were clustered into operational taxonomic units (OTUs) with average neighbor clustering employing a cutoff value of $10 \%$ sequence identity. For analyses of diversity and composition, an OTU definition of $\geq 97 \%$ sequence identity was employed.

Statistical analyses of sequence data. For the nonmetric multidimensional scaling (NMDS) analysis, pairwise Bray-Curtis distances (incorporating relative abundance) were calculated between samples and axes were calculated in the mothur software package. To test if the clustering between vegetated and SVD communities was significant, analyses of similarities statistics (ANOSIM) (Anderson and Walsh 2013) were performed within the mothur software package.

Significant differences in OTU relative abundance were determined using Welch's $t$ test with the Benjamin-Hochberg false discovery rate correction for multiple test corrections as implemented in the STAMP software package (Parks and Beiko 2010). The raw outputs from STAMP were imported into the R statistical software environment ( $R$ Core Team 2013) for data visualization. Relative abundance of OTUs was displayed on a logarithmic scale as this better represents the large variation in values as previously reported (Wang et al. 2016). All sequences generated in this study are available in the NCBI sequence read archive under the accession number SRP090554.

Greenhouse experiments. S. alterniflora was produced from seed collected from healthy plants at Hammonasset Beach State Park. Seed was surface-sterilized in $0.05 \% \mathrm{NaClO}$ for $1 \mathrm{~min}$, rinsed in sterile tap water three times, and placed in wet sand in trays on greenhouse benches. After 10 to 20 days, germinated seedlings were transplanted into 1:1 top soil/ProMix BX soilless potting mix in 36 cell trays and maintained for 4 to 6 weeks or 1 year for the transplants. Plants were kept well irrigated and fertilized once every 2 months with Miracle-Gro Water Soluble All Purpose Plant Food 24-8-16.

To assess whether the microbial communities in SVD affected sediments were deleterious to the growth of $S$. alterniflora, sediments were collected from the SVD sites at Hammonasset Beach State Park in October 2015. The top $10 \mathrm{~cm}$ of soil was removed with a spade, transported to the CAES, and sieved to remove large chunks of roots in the greenhouse. One half of the soil was autoclaved at $121^{\circ} \mathrm{C}$ for $1 \mathrm{~h}$ on two consecutive days. Both autoclaved and non-autoclaved sediments were each dispensed into 201 -liter plastic pots $(800 \mathrm{cc} / \mathrm{pot})$. To provide a non-marsh control soil, a 1:1 top soil (Agway Inc.) and Promix BX potting soil was also included.

Ten seeds of $S$. alterniflora were placed on the soil surface of 10 pots filled with each soil treatment and kept moist. The seeds were monitored for several weeks and the number of germinated seedlings was recorded. Ten replicate pots of each soil treatment were also planted with seedlings that were either 6 weeks old or mature 1 -year-old plants. Plants were rated for disease symptoms on a scale of 1 to 6 , where $1=$ healthy robust and $6=$ dead after 40,70 , and 80 days after planting. Disease progress was calculated using the area under the disease progress curve (AUDPC) and was calculated using the trapezoid rule 


$$
\mathrm{AUDPC}=\sum\left(Y_{i}+Y_{i+1}\right) / 2 \times\left(t_{i+1}-t_{i}\right)
$$

where $Y_{\mathrm{i}}=$ disease rating and $t_{\mathrm{i}}=$ time initial (Jeger and ViljanenRollinson 2001). On the 80th day, plants were harvested and all above-ground tissue was dried and weighed. Dry weight data were analyzed using analysis of variance and means were separated using Tukey's honestly significant difference test at $P=0.05$.

\section{RESULTS}

Sediment characteristics and sediment $\mathrm{CO}_{2}$ flux. Across the different measures of soil characteristics, only the sediment water content was found to significantly differ $(P=0.03)$ between the vegetated and SVD affected sites (Table 1). Additionally, $\mathrm{CO}_{2}$ flux, a measure of respiration, was $64 \%$ lower in the SVD affected sediments, a statistically significant difference $(P=0.05)$.

Sequence diversity. A total of 7,110,477 high quality sequences were generated in this study. The range of sequences per sample was 699 to 703,323. As differences in sequencing effort have been associated with skewed diversity estimates (Gihring et al. 2012), all of the sequence datasets were randomly subsampled to the size of the smallest library. Datasets with significantly smaller sequence recovery were omitted (Supplementary Table S1). This resulted in datasets of 17,799 sequences per sample. The average number of OTUs from the healthy vegetated soils and SVD affected soils were similar with 9,680 and 9,961 OTUs, respectively (Table 2). Additionally, there were no significant differences in the diversity estimates between the healthy and SVD affected soils. The estimated coverage of the datasets was in the range of $\sim 50 \%$, suggesting that with this sequencing effort, only a fraction of the total expected diversity of these samples was recovered.

Taxonomic composition of sediment bacterial communities. Relative abundance of the OTUs in the rarefied datasets was employed to test for compositional differences between the vegetated and SVD affected sediment communities by NMDS. The NMDS analysis showed clear independent clustering between the communities in vegetated versus SVD affected sediments (Fig. 2). This independent clustering was supported by ANOSIM statistics, which indicated a highly significant difference in the structure of the communities between vegetated and SVD affected sediments $(P<0.001)$ (Fig. 2).

OTU relative abundance data were used to identify taxonomic groups that were significantly different in relative abundance between the vegetated and SVD affected sediment communities (Fig. $3 \mathrm{~A})$. The shaded boxes in the upper panels of the figure are displayed such that they encompass OTUs with highly significant statistical results $(P \leq 0.001)$ and large changes in relative abundance (threefold difference in relative abundance) between SVD and vegetated sediments. Thus, these OTUs are those that show large and highly significant changes, presumably making these among the most biologically meaningful shifts associated with SVD affected sediments. These parameters identified 81 OTUs as significantly more abundant in the vegetated sediments compared with 67 OTUs more abundant in the SVD affected sediments (Fig. 3A). A full list of these OTUs is available in Supplementary Table S2.

Proteobacteria accounted for the majority of the differentially abundant OTUs, accounting for $43(53 \%)$ of the OTUs enriched in the vegetated sediments and $44(66 \%)$ of the OTUs significantly more abundant in the SVD sediments. A notable difference between the vegetated and SVD affected sediments was the relatively large proportion of Bacteroidetes that were more abundant in the vegetated sediments. Of the OTUs identified as more abundant in the

TABLE 2

Operational taxonomic units (OTUs) diversity in the sequence datasets

\begin{tabular}{lccc}
\hline Condition & OTUs $^{y}$ & Shannon's diversity $^{y}$ & Coverage $^{\mathrm{y}, \mathrm{z}}$ \\
\hline Vegetated & $9,680( \pm 617)$ & $8.6( \pm 0.2)$ & $53.8( \pm 3.2)$ \\
\hline SVD & $9,961( \pm 832)$ & $8.7( \pm 0.1)$ & $51.6( \pm 2.1)$ \\
\hline
\end{tabular}

y Calculated from the subsampled datasets. Values represent averages across the replicate datasets $(n=15$ for sudden vegetation dieback [SVD] and $n=18$ for vegetated) \pm standard deviation. None of the comparisons between the groups were found to be statistically significant by Student's $t$ test. The values for individual datasets are presented in Supplementary Table S1.

z Good's coverage (Good 1953).

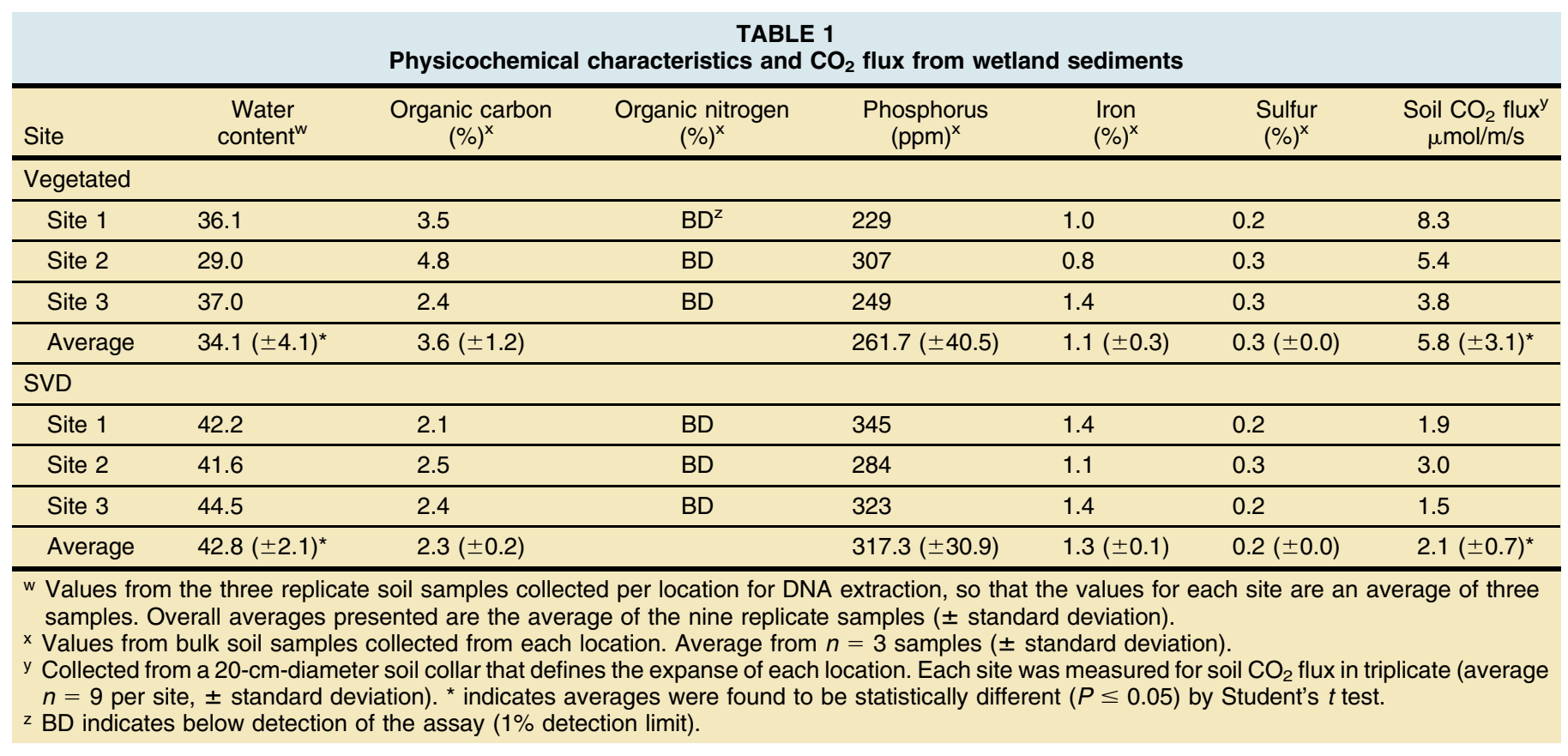


vegetated sediments, 27 (33\%) were Bacteroidetes compared with four $(6 \%)$ being more abundant in the SVD affected sediments (Fig. 3B). These observations suggest that vegetation loss may be associated with a decline of Bacteroidetes-related bacteria. The remaining OTUs belonged to 11 phyla or were not able to be classified. Individually, these groups made up a relatively small proportion of differentially abundant OTUs (Fig. 3B).

To characterize the differentially abundant OTUs at deeper taxonomic levels, the 10 OTUs showing the most significant changes in relative abundance (ranked by $P$ value) that were either more abundant in the vegetated or SVD affected sediments are displayed in Figures 4 and 5. Of the OTUs that were more abundant in the vegetated sediments, 7 of the 10 that were most significantly altered in relative abundance could not be classified past their phylum (Fig. 4). The remaining three OTUs belonged to the genera Flexithrix, Imtechella, and Lewinella, all within the phylum Bacteroidetes.

Within the OTUs more abundant in SVD affected sediments, only two were not classified to the genus level (Fig. 5). Of the 10 OTUs determined to be more abundant in SVD affected sediments, five were classified to the same genus, Desulfobulbus (phylum Proteobacteria) and two to the genus Acidiferrobacter (phylum Proteobacteria) (Fig. 5), suggesting the majority of the differentially abundant OTUs enriched in SVD affected sediments were close taxonomic relatives.

Germination and growth of S. alterniflora in SVD affected soils. The final objective of the study was to test if the alterations in the sediment bacterial communities could be associated with the initial onset of SVD or the inability of S. alterniflora to recolonize these sites after a dieback event. There was a trend toward lower germination rates in the seedlings in the natural sediment compared with autoclaved sediments, or the control soil, but the differences were not significant (Table 3). Seedlings had more yellowing, stunting, and less vigor in the autoclaved and the natural (nonautoclaved) SVD sediments than in the soil mix treatment, although the dry weights of the recovered plants did not significantly differ
(Table 3). Disease progress estimates (AUDPC) of transplants did not differ among transplant in the experiment, but plants grown in autoclaved SVD sediments were significantly larger than the soil mix control or natural sediments. Taken together, these data suggest, at most, there is a limited effect of the SVD sediment microbial community on the health of S. alterniflora, and the lack of mortality suggests that the indigenous sediment microbiota are not likely associated with the development of SVD.

\section{DISCUSSION}

The SVD affected sediments retained physicochemical characteristics similar to those of the vegetated sediments (Table 1), even after 10 years from the initial development of SVD. This matches observations of dieback sites in Georgia, which identified few changes in soil chemistry associated with SVD (Crawford and Stone 2014). The one notable difference between these studies is that sites in Georgia associated with SVD showed significantly lower water content than unvegetated sites, whereas the SVD sites in this study showed elevated water content (Table 1). This suggests that sediment interactions with SVD may differ between wetlands or with other site-specific characteristics.

The $\mathrm{CO}_{2}$ flux from SVD affected sediments, a measure of microbial respiration, was approximately $36 \%$ of that from vegetated sediments (Table 1). Similarly, other studies have recorded lower $\mathrm{CO}_{2}$ flux from unvegetated wetland patches (Bahlmann et al. 2015). $S$. alterniflora translocates carbon from the atmosphere to the subsurface, increasing microbial respiration and mineralization in the vicinity of roots (Hines et al. 1989). Thus, the removal of primary productivity and the associated carbon inputs predictably results in lower respiration rates in the SVD sites.

The bacterial communities displayed notable and significant differences in composition between the SVD affected and vegetated sediments (Fig. 2). One of the more pronounced differences between the bacterial communities between the vegetated and SVD affected

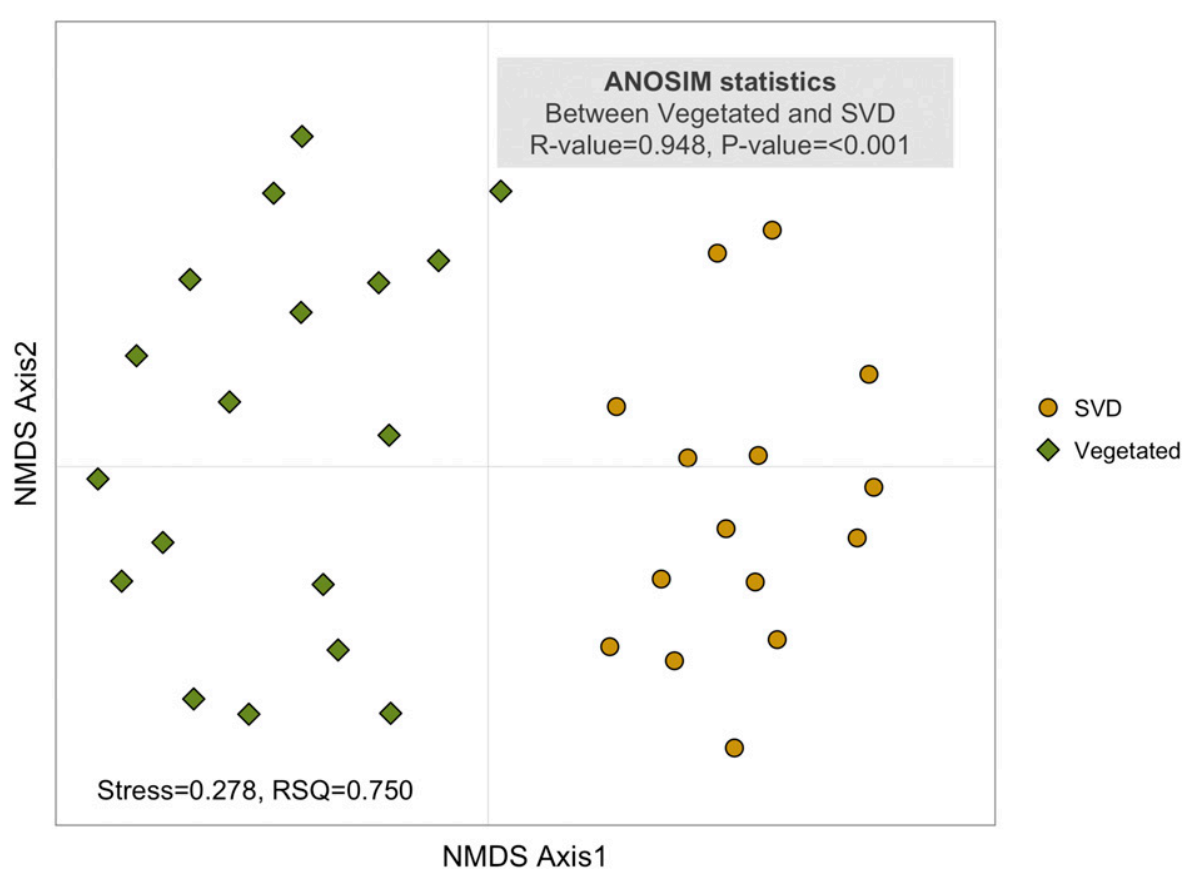

Fig. 2. Nonmetric multidimensional scaling (NMDS) analysis of operational taxonomic unit (OTU)-based clustering of sediment communities. Distances between datasets were determined using the rarefied raw count OTU data and calculated using the Bray-Curtis similarity metric incorporating OTU relative abundance. Results of the ANOSIM statistical test are indicated in the shaded panel. 
sites was the significant enrichment of OTUs belonging to the phylum Bacteroidetes in the vegetated sediments (Fig. 3B). This suggests a potential role for these organisms in the processing of the carbon produced by S. alterniflora. This is not the first study to detect a reduction of Bacteroidetes bacteria in association with vegetation loss. For example, in an arid grassland, experimental vegetation removal resulted in large significant decreases in the proportion of Bacteroidetes recovered from soils (McHugh and Schwartz 2015). Bacteroidetes relative abundance has also been positively correlated to carbon respiration rates (Fierer et al. 2007). Here we show that Bacteroidetes were more abundant in vegetated sediments, which also supported higher respiration rates (Table 1). Taken together, these observations support a strong role for the Bacteroidetes in saprotrophic carbon cycling in these coastal wetland sediments.

Among the OTUs identified as the most significantly enriched in the vegetated sediments, all but two belonged to the phylum Bacteroidetes (Fig. 4). Of the Bacteroidetes-related OTUs, three were classified to the genera Flexithrix, Imtechella, and Lewinella, all of which are aerobic, halotolerant, heterotrophs (Khan et al. 2007; Nakagawa et al. 2002; Surendra et al. 2012). A large proportion of the total of 81 OTUs that were identified as significantly more abundant in vegetated sediments (Fig. 3A) could not be classified to the genus level. In this respect, there is little that can be inferred from these taxonomic classifications. This suggests that coastal wetland sediments harbor uncharacterized bacteria, particularly within the phylum Bacteroidetes, which may be important to ecosystem function, specifically in processing carbon fixed by S. alterniflora.

In the SVD affected sediments, five of the ten most significantly enriched OTUs were within the genus Desulfobulbus, a group of anaerobic sulfate reducing bacteria (Castro et al. 2000; RooneyVarga et al. 1997). These observations point to an increased role of anoxic metabolism in the SVD affected sediments. Two factors could account for lower oxygen contents in SVD affected sites. First, our measurement of increased water loading in SVD affected sites, as the increased water content would be expected to decrease the movement of oxygen into sediment pore spaces. Secondly, $S$. alterniflora translocates oxygen from leaves to the roots, locally increasing oxygen content in subsurface sediments (Borum et al. 2005; Mendelssohn et al. 1981; Teal and Kanwisher 1966). In this
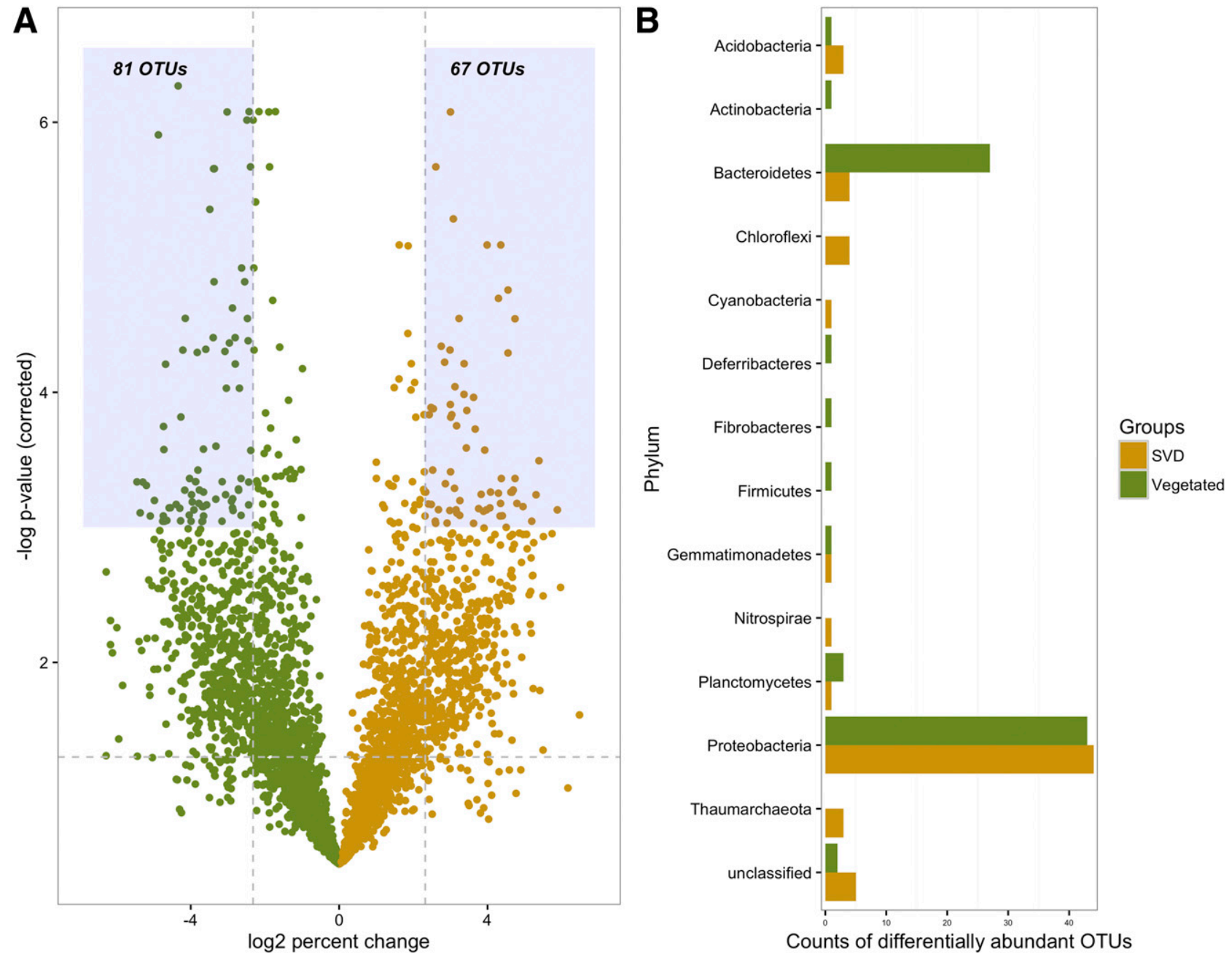

Fig. 3. Differentially abundant operational taxonomic units (OTUs) between vegetated and sudden vegetation dieback (SVD) affected sediments. A, Volcano plot displaying statistical significance versus fold change in relative abundance of each OTU in the dataset. The $P$ values are displayed as the negative log10 value so that lower $P$ values appear higher on the $y$ axis. The horizontal dashed line denotes a $P$ value of 0.05 , and the vertical lines denote a threefold change in relative abundance. The blue shaded boxes encompass OTUs with a $P$ value of $\leq 0.001$ and a threefold change in relative abundance, and therefore represent OTUs with highly significant and large shifts in relative abundance between vegetated and SVD affected sediments. B, Phylum level classification of OTUs identified as significantly different in panel A (OTUs within shaded boxes). Bars represent the number of OTUs identified within each phylum. 


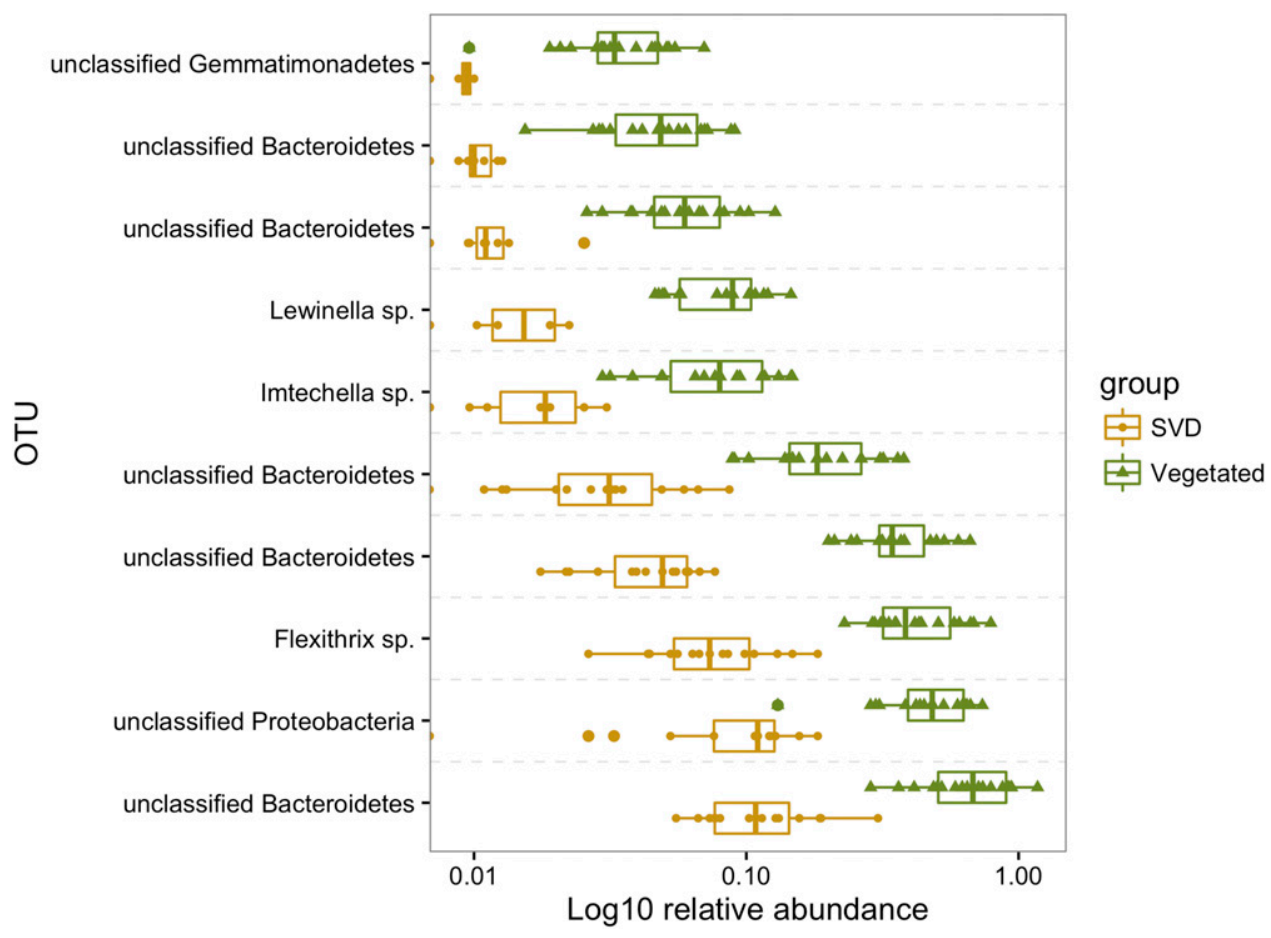

Fig. 4. Operational taxonomic units (OTUs) enriched in vegetated sediments. Boxplot of the 10 OTUs identified as most significantly enriched in vegetated sediments (ranked by $P$ value). The vertical lines represent the mean, with the boxes encompassing the 25th and 75 th percentiles of the data, the whiskers the 5th and 95th percentiles, and outliers represented by dots outside the boxes and whiskers. Each OTU was classified to the genus level, unless indicated by unclassified, in which case the deepest taxonomic assignment is indicated. OTUs were classified using the consensus of sequences that made up the OTU. A full list of differentially abundant OTUs is presented in Supplementary Table S2.

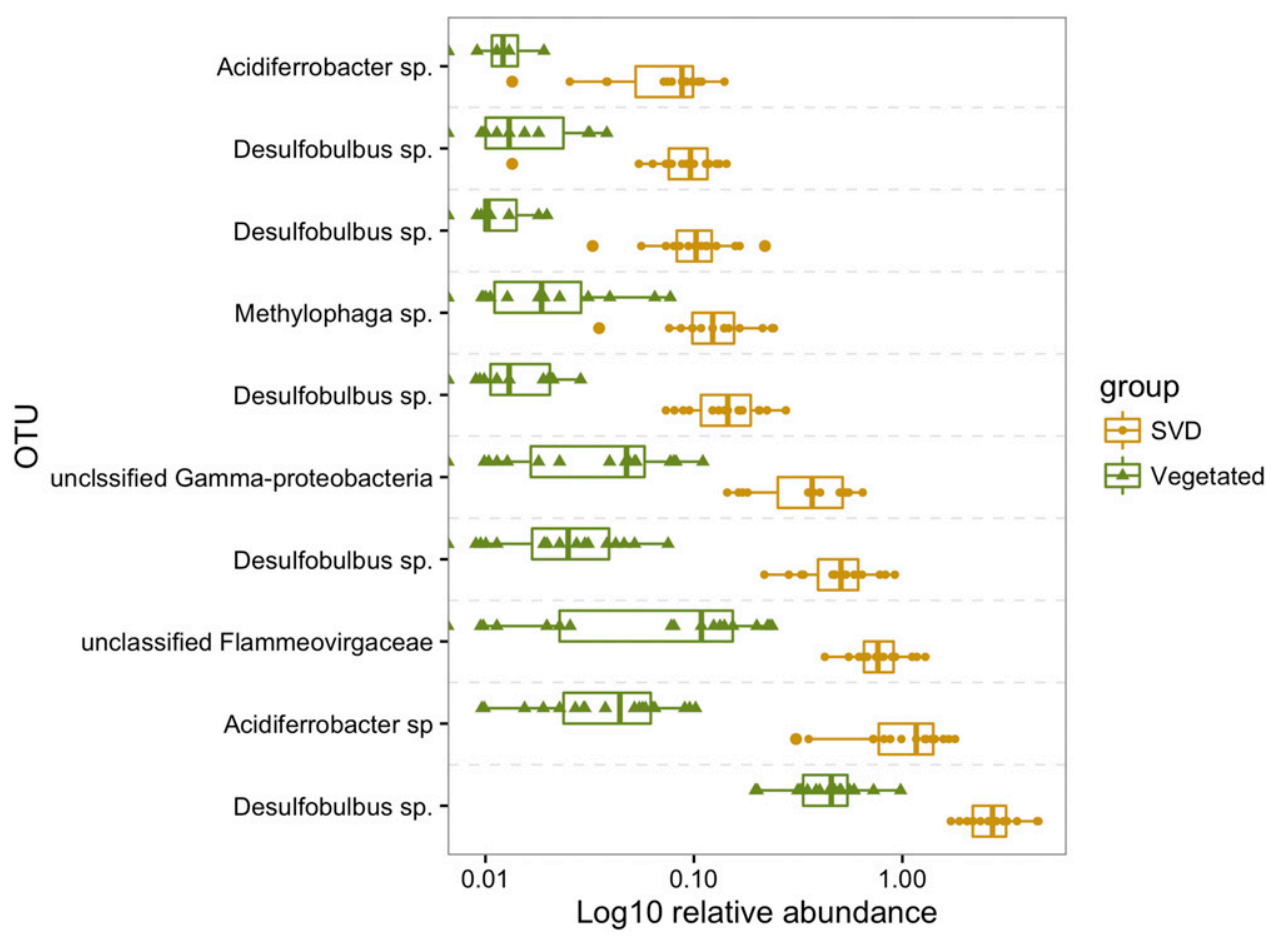

Fig. 5. Operational taxonomic units (OTUs) enriched in sudden vegetation dieback (SVD) affected sediments. Boxplot of the 10 OTUs identified as the most significantly enriched in SVD affected sediments (ranked by $P$ value). The vertical lines represent the mean, with the boxes encompassing the 25th and 75th percentiles of the data, the whiskers the 5th and 95th percentiles, and outliers represented by dots outside the boxes and whiskers. Each OTU was classified to the genus level, unless indicated by unclassified, in which case the deepest taxonomic assignment is indicated. OTUs were classified using the consensus of sequences that made up the OTU. A full list of differentially abundant OTUs is presented in Supplementary Table S2. 
respect, the loss of vegetation due to SVD would also cut off this source of oxygenation. Thus, the response of the microbial community to SVD reflects a shift toward more anoxic metabolism. Despite the potential for increased anaerobic metabolism in the SVD affected sediments, no methanogen related OTUs were identified as being significantly enriched in the SVD sites (Fig. 3B), suggesting methanogenesis did not significantly increase in the SVD sediments. Future work specifically investigating methane cycling should lead to a more complete view of the carbon processing in these coastal wetland sediments.

An additional two OTUs identified as significantly enriched in association with SVD were classified to the genus Acidiferrobacter (Fig. 5), primarily iron-oxidizing bacteria also capable of sulfur oxidation (Hallberg et al. 2011; King and Garey 1999). As Desulfobulbus and Acidiferrobacter employ inorganic compounds as electron acceptors and donors, respectively, this may reflect a reduced availability of organic carbon for metabolism, further implicating the role of $S$. alterniflora in maintaining the sediment microbial communities.

Other groups, such as the Cyanobacteria and Chloroflexi, showed an increase in abundance in SVD affected sediments (Fig. 3B). This could indicate an increased potential for microbial mediated primary productivity, perhaps reflecting an ecological replacement of the vegetation lost to SVD.

Finally, to investigate if the alterations in the microbial community in sediments associated with SVD could affect $S$. alterniflora development or health, greenhouse growth experiments were performed. Although minor differences in growth and or survival of $S$. alterniflora were observed in the experiment, altering the SVD microbial community through autoclaving had a slight positive effect on the dry weight recovery of seedlings and of transplants, but an increase in AUDPC of seedlings (Table 3). These differences might explained by the autoclaving process. Autoclaving soils can have positive or negative effect on plants growth by release beneficial and/ or toxic metabolites (Berns et al. 2008). The results presented here suggest that autoclaved soils have differing effects on seedlings versus mature transplants, suggesting potential differences in nutrients or metabolite requirements between different developmental stages of S. alterniflora. More importantly, the natural SVD sediments were not inhibitory to $S$. alterniflora germination or growth (Table 3), which suggests that alterations in nutrients or the sediment microbial community that occur following an SVD event are not likely associated with the plant mortality or the failure of

\section{TABLE 3}

Growth of Spartina alterniflora in greenhouse experiments ${ }^{x}$

\begin{tabular}{|c|c|c|c|}
\hline Sample & Germination $^{y}$ & AUDPC $^{z}$ & Dry weight (g) \\
\hline \multicolumn{4}{|l|}{ Seedlings } \\
\hline $1: 1$ soil/mix control & $9 a$ & $79.3 \mathrm{a}$ & $0.27 \mathrm{a}$ \\
\hline Autoclaved SVD soil & $8 a$ & $151.4 \mathrm{~b}$ & $0.26 \mathrm{a}$ \\
\hline Natural SVD soil & $6 a$ & $154.4 \mathrm{~b}$ & $0.13 \mathrm{a}$ \\
\hline \multicolumn{4}{|l|}{ Transplants } \\
\hline $1: 1$ soil/mix control & - & $91.6 \mathrm{a}$ & $4.46 \mathrm{a}$ \\
\hline Autoclaved SVD soil & - & $58.6 \mathrm{a}$ & $8.13 b$ \\
\hline Natural SVD soil & - & $81.6 \mathrm{a}$ & $5.07 \mathrm{a}$ \\
\hline
\end{tabular}

S. alterniflora to recolonize the unvegetated patches. The limited role of sediment microbes in SVD is further supported by reports of successful transplants between vegetated and SVD affected sites in natural wetlands, although reduced growth in transplanted grasses is often reported (Alber et al. 2008). Currently, factors such as the invasion of the crab Sesarma reticulatum are thought to play a role in maintaining SVD (Elmer et al. 2013). Future research investigating the causes, consequences, maintenance, and restoration of SVD affected wetlands needs to be pursued, while incorporating the multiple trophic levels that operate in coastal wetlands.

In summary, the large scale disturbance of coastal wetlands induced by SVD translate into a restructuring of the sediment bacterial communities. The shifts in the bacterial communities point to large scale changes in the metabolic pathways and ecosystem process rates related to carbon cycling. In this respect, the development of SVD may represent a natural experiment in which to gain a mechanistic understanding of how plants and their phytobiome interact to affect how carbon is cycled and stored in wetland sediments. Furthermore, understanding the causes and consequences of SVD needs to be pursued to inform carbon budgets of wetlands experiencing SVD and developing effective management and conservation strategies to protect these valuable ecosystems.

\section{ACKNOWLEDGMENTS}

B. Steven would like to acknowledge funding from National Institute of Food and Agriculture grant 1006211. We thank H. Alves, Park and Recreation Supervisor for Hammonasset Beach State Park for site access, and R. Huntley for assistance with field collections.

\section{LITERATURE CITED}

Alber, M., Swenson, E. M., Adamowicz, S. C., and Mendelssohn, I. A. 2008. Salt marsh dieback: An overview of recent events in the U.S. Estuar. Coast. Shelf Sci. 80:1-11.

Anderson, M. J., and Walsh, D. C. I. 2013. PERMANOVA, ANOSIM, and the Mantel test in the face of heterogeneous dispersions: What null hypothesis are you testing? Ecol. Monogr. 83:557-574.

Bahlmann, E., Weinberg, I., Lavrič, J. V., Eckhardt, T., Michaelis, W., Santos, R., and Seifert, R. 2015. Tidal controls on trace gas dynamics in a seagrass meadow of the Ria Formosa lagoon (southern Portugal). Biogeosciences 12:1683-1696.

Berns, A. E., Philipp, H., Narres, H.-D., Burauel, P., Vereecken, H., and Tappe, W. 2008. Effect of gamma-sterilization and autoclaving on soil organic matter structure as studied by solid state NMR, UV and fluorescence spectroscopy. Eur. J. Soil Sci. 59:540-550.

Bertness, M. D., Crain, C., Holdredge, C., and Sala, N. 2008. Eutrophication and consumer control of New England salt marsh. Conserv. Biol. 22: 131-139.

Blodau, C. 2002. Carbon cycling in peatlands-A review of processes and controls. Environ. Rev. 10:111-134.

Borum, J., Pedersen, O., Greve, T. M., Frankovich, T. A., Zieman, J. C., Fourqurean, J. W., and Madden, C. J. 2005. The potential role of plant oxygen and sulphide dynamics in die-off events of the tropical seagrass, Thalassia testudinum. J. Ecol. 93:148-158.

Brevik, E. C., and Homburg, J. A. 2004. A 5000 year record of carbon sequestration from a coastal lagoon and wetland complex, Southern California, USA. Catena 57:221-232.

Bu, N., Qu, J., Li, Z., Li, G., Zhao, H., Zhao, B., Li, B., Chen, J., and Fang, C. 2015. Effects of Spartina alterniflora invasion on soil respiration in the Yangtze River Estuary, China. PLoS One 10:e0121571.

Castro, H. F., Williams, N. H., and Ogram, A. 2000. Phylogeny of sulfatereducing bacteria. FEMS Microbiol. Ecol. 31:1-9.

Choi, Y., and Wang, Y. 2004. Dynamics of carbon sequestration in a coastal wetland using radiocarbon measurements. Global Biogeochem. Cycles 18: GB4016.

Crawford, J. T., and Stone, A. G. 2014. Relationships between soil composition and Spartina alterniflora dieback in an Atlantic salt marsh. Wetlands 35: 13-20. 
Edgar, R. C., Haas, B. J., Clemente, J. C., Quince, C., and Knight, R. 2011 UCHIME improves sensitivity and speed of chimera detection. Bioinformatics 27:2194-2200.

Elmer, W. H. 2014. A tripartite interaction between Spartina alterniflora, Fusarium palustre, and the purple marsh crab (Sesarma reticulatum) contributes to sudden vegetation dieback of salt marshes in New England. Phytopathology 104:1070-1077.

Elmer, W. H. 2016. Pathogenic microfungi associated with spartina in salt marshes. Pages 615-630 in: Biology of Microfungi. D.-W. Li, ed. Springer International Publishing.

Elmer, W. H., LaMondia, J. A., and Caruso, F. L. 2012. Association between Fusarium spp. on Spartina alterniflora and dieback sites in Connecticut and Massachusetts. Estuaries Coasts 35:436-444.

Elmer, W. H., Useman, S., Schneider, R. W., Marra, R. E., LaMondia, J. A., Mendelssohn, I. A., Jimenez-Gasco, M. M., and Caruso, F. L. 2013. Sudden vegetation dieback in Atlantic and Gulf Coast salt marshes. Plant Dis. 97: 436-445.

Fierer, N., Bradford, M. A., and Jackson, R. B. 2007. Toward an ecological classification of soil bacteria. Ecology 88:1354-1364.

Gihring, T. M., Green, S. J., and Schadt, C. W. 2012. Massively parallel rRNA gene sequencing exacerbates the potential for biased community diversity comparisons due to variable library sizes: Pyrosequencing exacerbates sample size bias. Environ. Microbiol. 14:285-290.

Good, I. J. 1953. The population frequencies of species and the estimation of population parameters. Biometrika 40:237-264.

Hallberg, K. B., Hedrich, S., and Johnson, D. B. 2011. Acidiferrobacter thiooxydans, gen. nov. sp. nov.; an acidophilic, thermo-tolerant, facultatively anaerobic iron- and sulfur-oxidizer of the family Ectothiorhodospiraceae. Extremophiles 15:271-279.

Herr, D., Pidgeon, E., and Laffoley, D.2012. Blue carbon policy framework 2.0: Based on the discussion of the International Blue Carbon Policy Working Group. IUCN, Gland, Switzerland.

Hines, M. E., Knollmeyer, S. L., and Tugel, J. B. 1989. Sulfate reduction and other sedimentary biogeochemistry in a northern New England salt marsh. Limnol. Oceanogr. 34:578-590.

Hopkinson, C. S., Cai, W.-J., and Hu, X. 2012. Carbon sequestration in wetland dominated coastal systems-A global sink of rapidly diminishing magnitude. Curr. Opin. Environ. Sustain. 4:186-194.

Howes, B. L., Dacey, J. W. H., and Teal, J. M. 1985. Annual carbon mineralization and belowground production of Spartina alterniflora in a New England salt marsh. Ecology 66:595-605.

Jeger, M. J., and Viljanen-Rollinson, S. L. H. 2001. The use of the area under the disease-progress curve (AUDPC) to assess quantitative disease resistance in crop cultivars. Theor. Appl. Genet. 102:32-40.

Kennedy, H., Beggins, J., Duarte, C. M., Fourqurean, J. W., Holmer, M., Marbà, N., and Middleburg, J. J. 2010. Seagrass sediments as a global carbon sink: Isotopic constraints. Global Biogeochem. Cycles 24:GB4026.

Khan, S. T., Fukunaga, Y., Nakagawa, Y., and Harayama, S. 2007. Emended descriptions of the genus Lewinella and of Lewinella cohaerens, Lewinella nigricans and Lewinella persica, and description of Lewinella lutea sp. nov. and Lewinella marina sp. nov. Int. J. Syst. Evol. Microbiol. 57:2946-2951.

King, G. M., and Garey, M. A. 1999. Ferric iron reduction by bacteria associated with the roots of freshwater and marine macrophytes. Appl. Environ. Microbiol. 65:4393-4398.

Lal, R. 2004. Soil carbon sequestration to mitigate climate change. Geoderma 123:1-22.

Liao, C., Luo, Y., Jiang, L., Zhou, X., Wu, X., Fang, C., Chen, J., and Li, B. 2007. Invasion of Spartina alterniflora enhanced ecosystem carbon and nitrogen stocks in the Yangtze Estuary, China. Ecosystems (N. Y.) 10:1351-1361.

Loiacono, F., De Marco, A., Dicoladonato, G., Gadaleta, M. V., and Durmishi, C. 2011. Recent sedimentation in the Vlora Gulf: Grain size and mineralogical analyses from some cores in a E-W transect. J. Coast. Res. 270:17-25.
McHugh, T. A., and Schwartz, E. 2015. Changes in plant community composition and reduced precipitation have limited effects on the structure of soil bacterial and fungal communities present in a semiarid grassland. Plant Soil 388:175-186.

McKee, K. L., Cahoon, D. R., and Feller, I. C. 2007. Caribbean mangroves adjust to rising sea level through biotic controls on change in soil elevation. Glob. Ecol. Biogeogr. 16:545-556.

McKee, K. L., Mendelssohn, I. A., and Materne, M. D. 2004. Acute salt marsh dieback in the Mississippi River deltaic plain: A drought-induced phenomenon? Glob. Ecol. Biogeogr. 13:65-73.

Mcleod, E., Chmura, G. L., Bouillon, S., Salm, R., Björk, M., Duarte, C. M., Lovelock, C. E., Schlesinger, W. H., and Silliman, B R. 2011. A blueprint for blue carbon: Toward an improved understanding of the role of vegetated coastal habitats in sequestering $\mathrm{CO}_{2}$. Front. Ecol. Environ. 9:552-560.

Mendelssohn, I. A., Mckee, K. L., and Patrick, W. H. 1981. Oxygen deficiency in Spartina alterniflora roots: Metabolic adaptation to anoxia. Science 214: 439-441.

Nakagawa, Y., Sakane, T., Suzuki, M., and Hatano, K. 2002. Phylogenetic structure of the genera Flexibacter, Flexithrix, and Microscilla deduced from 16S rRNA sequence analysis. J. Gen. Appl. Microbiol. 48:155-165

Nellemann,C., and Cocoran,E. (eds.) 2009. Blue carbon: The role of healthy oceans in binding carbon: A rapid response assessment. UNEP/Earthprint.

Parks, D. H., and Beiko, R. G. 2010. Identifying biologically relevant differences between metagenomic communities. Bioinformatics 26:715-721.

Picek, T., Čížková, H., and Dušek, J. 2007. Greenhouse gas emissions from a constructed wetland-Plants as important sources of carbon. Ecol. Eng. 31:98-106.

R Core Team. 2013. R: A language and environment for statistical computing. Vienna, Austria. Published online. http://www.R-project.org/

Rooney-Varga, J. N., Devereux, R., Evans, R. S., and Hines, M. E. 1997. Seasonal changes in the relative abundance of uncultivated sulfate-reducing bacteria in a salt marsh sediment and in the rhizosphere of Spartina alterniflora. Appl. Environ. Microbiol. 63:3895-3901.

Schimel, D. S. 1995. Terrestrial ecosystems and the carbon cycle. Glob. Change Biol. 1:77-91.

Schloss, P. D., Westcott, S. L., Ryabin, T., Hall, J. R., Hartmann, M., Hollister, E. B., Lesniewski, R. A., Oakley, B. B., Parks, D. H., Robinson, C. J., Sahl, J. W., Stres, B., Thallinger, G. G., Van Horn, D. J., and Weber, C. F. 2009. Introducing mothur: Open-source, platform-independent, community-supported software for describing and comparing microbial communities. Appl. Environ. Microbiol. 75:7537-7541.

Schultz, R. A., Anisfeld, S. C., and Hill, T. D. 2016. Submergence and herbivory as divergent causes of marsh loss in Long Island Sound. Estuaries Coasts 39:1367-1375

Spivak, A. C., and Reeve, J. 2015. Rapid cycling of recently fixed carbon in a Spartina alterniflora system: a stable isotope tracer experiment. Biogeochemistry 125:97-114.

Surendra, V., Bhawana, P., Suresh, K., Srinivas, T. N. R., and Kumar, P. A. 2012. Imtechella halotolerans gen. nov., sp. nov., a member of the family Flavobacteriaceae isolated from estuarine water. Int. J. Syst. Evol. Microbiol. 62:2624-2630.

Teal, J. M., and Kanwisher, J. W. 1966. Gas transport in the marsh grass, Spartina alterniflora. J. Exp. Bot. 17:355-361.

Wang, F., Kaplan, J. L., Gold, B. D., Bhasin, M. K., Ward, N. L., Kellermayer, R., Kirschner, B. S., Heyman, M. B., Dowd, S. E., Cox, S. B., Dogan, H., Steven, B., Ferry, G. D., Cohen, S. A., Baldassano, R. N., Moran, C. J., Garnett, E. A., Drake, L., Out, H. H., Mirny, L., Libermann, T. A., Winter, H. S., and Korolev, K. S. 2016. Detecting microbial dysbiosis associated with pediatric Crohn disease despite the high variability of the gut microbiota. Cell Reports 14:945-955. 